\title{
Symmetrically coupled higher-order nonlinear Schrödinger equations: singularity analysis and integrability
}

\author{
S. Yu. Sakovich $\oplus$ and Takayuki Tsuchida田 \\ $\dagger$ Institute of Physics, National Academy of Sciences, P.O. 72, Minsk, Belarus \\ $\ddagger$ Department of Physics, Graduate School of Science, University of Tokyo, Hongo \\ 7-3-1, Bunkyo-ku, Tokyo 113-0033, Japan
}

\begin{abstract}
The integrability of a system of two symmetrically coupled higher-order nonlinear Schrödinger equations with parameter coefficients is tested by means of the singularity analysis. It is proven that the system passes the Painleve test for integrability only in ten distinct cases, of which two are new. For one of the new cases, a Lax pair and a multi-field generalization are obtained; for the other one, the equations of the system are uncoupled by a nonlinear transformation.
\end{abstract}

$\dagger$ E-mail: sakovich@dragon.bas-net.by

$\ddagger$ E-mail: tsuchida@monet.phys.s.u-tokyo.ac.jp 


\section{Introduction}

The nonlinear Schrödinger (NLS) equation, which describes the time evolution of slowly varying envelope, is encountered in various branches of physics and known to be fundamental. In deriving the NLS equation as the envelope equation, we neglect higher-order terms under appropriate physical assumptions. However, due to recent developments in optical technology, higher-order corrections to the NLS equation have become necessary and important. Kodama and Hasegawa [1, 2] proposed the higherorder NLS (HONLS) equation,

$$
q_{t}=h q_{x x x}+a q \bar{q} q_{x}+b q^{2} \bar{q}_{x}+\mathrm{i}\left(s q_{x x}+f q^{2} \bar{q}\right)
$$

which describes ultra-short pulse propagation in optical fibers, including higher-order effects such as higher-order dispersion, self-steeping and delayed Raman response. Here the bar denotes the complex conjugation, $\bar{q}=q^{*}$. For simplicity, we assume that the parameters $h, a, b, s, f$ are real and satisfy the conditions, $a^{2}+b^{2} \neq 0, s \neq 0$ if $h=0$. Integrable cases of the HONLS equation (1.1) attract both theoretical and experimental interest because they support a variety of exact solutions and the initial-value problem is solvable. To date, there are four known integrable cases of the HONLS equation (1.1) which are solvable via the inverse scattering method:

$$
\begin{array}{llll}
h \neq 0 & a \neq 0 & b=0 & f=s a /(3 h) ; \\
h \neq 0 & a \neq 0 & b=a / 3 & f=2 s a /(9 h) ; \\
h=0 & s \neq 0 & a \neq 0 & b=0 \\
h=0 & s \neq 0 & a \neq 0 & b=a / 2 .
\end{array}
$$

The cases (1.2)-(1.5) of the HONLS equation (1.1) are called, respectively, the Hirota equation [3], the Sasa-Satsuma equation [4, 5], the Chen-Lee-Liu equation [6] and the Kaup-Newell equation [7]. The Painlevé analysis of the HONLS equation, which has been carried out by a number of authors [8, 9, 10, 11, 12, strongly indicates that eq. (1.1) is integrable only in the four cases (1.2)-(1.5). The study based on prolongation structures leads to the same indication [13.

To describe two pulses copropagating in optical fibers, we need to consider a two-component generalization of the single-component propagation equation [14]. For this purpose, we consider the following system of two symmetrically coupled HONLS equations:

$$
\begin{aligned}
& q_{t}=h q_{x x x}+a q \bar{q} q_{x}+b q^{2} \bar{q}_{x}+c r \bar{r} q_{x}+d q \bar{r} r_{x}+e q r \bar{r}_{x}+\mathrm{i}\left(s q_{x x}+f q^{2} \bar{q}+g q r \bar{r}\right) \\
& r_{t}=h r_{x x x}+a r \bar{r} r_{x}+b r^{2} \bar{r}_{x}+c q \bar{q} r_{x}+d r \bar{q} q_{x}+e r q \bar{q}_{x}+\mathrm{i}\left(s r_{x x}+f r^{2} \bar{r}+g r q \bar{q}\right)
\end{aligned}
$$

where $h, a, b, c, d, e, s, f, g$ are real parameters and the bar denotes the complex conjugation. In fact, we can assume more general conditions $\bar{q}= \pm q^{*}, \bar{r}= \pm r^{*}$ with the double signs being arbitrary. This makes no difference in the following analysis. We impose the conditions, $a^{2}+b^{2}+c^{2}+d^{2}+e^{2} \neq 0, c^{2}+d^{2}+e^{2}+g^{2} \neq 0, s \neq 0$ if 
$h=0$, on the parameters so that the system (1.6) is not uncoupled and includes linear dispersion and higher-order nonlinearity. The system (1.6) is a natural generalization of the HONLS equation (1.1) to a two-component system, which is invariant under any of the transformations, $q \leftrightarrow r, q \rightarrow \mathrm{e}^{\mathrm{i} \alpha} q$ ( $\alpha$ : real), $r \rightarrow \mathrm{e}^{\mathrm{i} \beta} r(\beta$ : real).

In this paper, we study the system of coupled HONLS equations (1.6) by means of the Painlevé analysis. Similar attempts have been performed for coupled NLS equations without higher-order terms [15, 16]. However, on the Painlevé analysis of coupled HONLS equations, only a few papers of academic significance have appeared [17, 18], where the Painlevé test has been applied to only integrable cases or a rather restricted class of equations, compared with our general form (1.6). By using the Painlevé test, we exhaustively obtain the integrability conditions on the parameters in the coupled HONLS equations (1.6) for the first time.

The paper consists of the following. In section 2, we perform the singularity analysis of (1.6). It is proven that the system (1.6) passes the Painlevé test for integrability only in the following ten distinct cases:

$$
\begin{array}{lll}
h \neq 0 & a \neq 0 & b=0 \quad c=d=e=a \quad f=g=s a /(3 h) \\
h \neq 0 & a \neq 0 & b=d=g=0 \quad c=e=a / 2 \quad f=s a /(3 h) \\
h \neq 0 & a \neq 0 & b=d=e=0 \quad c=a \quad f=g=s a /(3 h) \\
h \neq 0 & a \neq 0 & b=e=0 \quad c=d=a / 2 \quad f=g=s a /(3 h) \\
h \neq 0 & a \neq 0 & b=d=e=a / 3 \quad c=2 a / 3 \quad f=g=2 s a /(9 h) \\
h=0 & s \neq 0 \quad a \neq 0 \quad b=d=e=0 \quad c=a \quad g=f \\
h=0 & s \neq 0 \quad a \neq 0 \quad b=c=d=e=a / 2 \quad g=f \\
h=0 & s \neq 0 \quad a \neq 0 \quad b=c=e=0 \quad d=a \quad g=f \\
h=0 & s \neq 0 \quad a \neq 0 \quad b=e=a / 2 \quad c=a \quad d=0 \quad g=f \\
h=0 & s \neq 0 \quad a \neq 0 \quad b=d=0 \quad c=e=a \quad g=0 .
\end{array}
$$

In section 3, we show that the system (1.6) is integrable in the Lax sense in all the cases (1.7)-(1.16). The integrability of (1.9)-(1.15) has already been studied in the literature. The case (1.8) turns out to be related to (1.10). For the case (1.7), we construct a corresponding $4 \times 4$ Lax pair and propose a multi-field generalization. For the case (1.16), we obtain a nonlinear transformation, which changes the system (1.6) into two independent Chen-Lee-Liu equations. The last section, section $\mathbb{6}$, is devoted to concluding remarks. 


\section{Singularity analysis}

\subsection{Preliminaries}

Let us apply the Painlevé test for integrability to the system (1.6). It would be better to say "the Kovalevskaya test" [19], because we, following the Weiss-Kruskal algorithm of the singularity analysis [20, 21], select only those cases of the tested system, in which its general solution admits series expansions of Laurent type.

With respect to $q, \bar{q}, r, \bar{r}$, which should be considered as mutually independent, the system (11.6) is a normal system of four PDEs, of total order twelve if $h \neq 0$ or eight if $h=0, s \neq 0$. A hypersurface $\phi(x, t)=0$ is non-characteristic for (1.6) if $\phi_{x} \neq 0$, and we set $\phi_{x}=1$. The substitution of the expansions

$$
\begin{aligned}
& q=q_{0}(t) \phi^{\alpha}+\cdots+q_{n}(t) \phi^{n+\alpha}+\cdots \\
& \bar{q}=\bar{q}_{0}(t) \phi^{\beta}+\cdots+\bar{q}_{n}(t) \phi^{n+\beta}+\cdots \\
& r=r_{0}(t) \phi^{\gamma}+\cdots+r_{n}(t) \phi^{n+\gamma}+\cdots \\
& \bar{r}=\bar{r}_{0}(t) \phi^{\delta}+\cdots+\bar{r}_{n}(t) \phi^{n+\delta}+\cdots
\end{aligned}
$$

(the bar does not mean the complex conjugation now) into the system (1.6) determines the branches, i.e. admissible choices of $\alpha, \beta, \gamma, \delta$ and $q_{0}, \bar{q}_{0}, r_{0}, \bar{r}_{0}$, as well as the positions $n$ of resonances for those branches.

We require that the system (1.6) admits a singular generic branch, where the exponents $\alpha, \beta, \gamma, \delta$ are integer and at least one of them is negative, the number of resonances is equal to the total order of the system, all the resonances but one lie in nonnegative integer positions, and the recursion relations for the coefficients of (2.1) are consistent at the resonances. The singular generic branches are studied in sections 2.2 and 2.3 for $h \neq 0$ and $h=0$, respectively; other branches are concisely considered in section 2.4. Computations are done by means of the Mathematica system [22], and we omit inessential details for this reason.

\subsection{Singular generic branches: $h \neq 0$}

When $h \neq 0$, we set $h=1$ without loss of generality. The consideration of dominant terms of (1.6) leads us to the following two cases to be studied separately: $\alpha+\beta=\gamma+\delta=-2$ and $\alpha+\beta=-2, \gamma+\delta>-2$ (for the reason of symmetry, we omit $\alpha+\beta>-2, \gamma+\delta=-2)$.

2.2.1. Case $\alpha+\beta=\gamma+\delta=-2$ In this case, we have two possibilities: either two or three of the resonances lie in the position $n=0$.

Two resonances at $\boldsymbol{n}=\mathbf{0}$. If we set $\alpha \neq \beta$ or $\gamma \neq \delta$, then the positions of four resonances are $n=-1,0,0,4$, and there are 54 distinct cases of admissible positions for other eight resonances. In none of the 54 cases, however, $\alpha, \beta, \gamma, \delta$ are integer simultaneously. 
Therefore we choose $\alpha=\beta=\gamma=\delta=-1$. Then we find from (1.6) and (2.1) that $q_{0} \bar{q}_{0}=r_{0} \bar{r}_{0}=$ constant $\neq 0$ (we set constant $=1$ w.l.g.), and that

$$
\begin{gathered}
a=-6-b-c-d-e \\
(n+1) n^{2}(n-3)(n-4)\left(n^{2}-6 n-2 b-2 d+5\right)\left(n^{2}-6 n-2 b-2 e+5\right) \times \\
\left(n^{3}-6 n^{2}+(5-2 d-2 e) n+4(c+d+e+3)\right)=0 .
\end{gathered}
$$

Five resonances lie in the positions $n=-1,0,0,3,4$, and, denoting the positions of other seven resonances as $n_{1}, n_{2}, \ldots, n_{7}$, we find from (2.3) that

$$
\begin{aligned}
& n_{2}=6-n_{1} \quad n_{4}=6-n_{3} \quad n_{7}=6-n_{5}-n_{6} \\
& d=\frac{1}{2}\left(5-2 b-6 n_{1}+n_{1}^{2}\right) \quad e=\frac{1}{2}\left(5-2 b-6 n_{3}+n_{3}^{2}\right) \\
& b=\frac{1}{4}\left(5-6 n_{1}+n_{1}^{2}-6 n_{3}+n_{3}^{2}+6 n_{5}-n_{5}^{2}+6 n_{6}-n_{6}^{2}-n_{5} n_{6}\right) \\
& c=\frac{1}{4}\left(-22+12 n_{5}-2 n_{5}^{2}+12 n_{6}-2 n_{6}^{2}-8 n_{5} n_{6}+n_{5}^{2} n_{6}+n_{5} n_{6}^{2}\right) .
\end{aligned}
$$

Taking into account the admissible multiplicity of the resonances, we have 23 distinct cases of their positions. In 22 of those cases, however, the recursion relations for the coefficients of (2.1) turn out to be inconsistent at the resonances (more details can be found in [23]). The only good case is $n_{1}=2, n_{3}=2, n_{5}=1, n_{6}=2$, when $n=-1,0,0,1,2,2,2,3,3,4,4,4, a=c=d=e=-3 / 2$ and $b=0$ due to (2.4) and (2.2). In this case, we have to set $f=g=-s / 2$ for the recursion relations to become consistent at $n=2,3$. Up to a scale transformation of variables, this is the case (1.7) of the system (1.6).

Three resonances at $\boldsymbol{n}=\mathbf{0}$. In this case, resonances can lie in admissible positions only if $q_{0} \bar{q}_{0}+r_{0} \bar{r}_{0}=$ constant (we set constant $=1$ w.l.g.) and

$\alpha=\beta=\gamma=\delta=-1 \quad a=-6-b \quad c=-6-d-e \quad(b-d)(b-e)=0$.

If $b=d, e \neq d$, we have

$$
(n+1) n^{3}(n-3)(n-4)\left(n^{2}-6 n+5-2 d\right)\left(n^{2}-6 n+5-d-e\right)^{2}=0
$$

i.e. six resonances lie in the positions $n=-1,0,0,0,3,4$. Denoting the positions of other six resonances as $n_{1}, n_{2}, \ldots, n_{6}$, we obtain

$$
\begin{aligned}
& n_{2}=6-n_{1} \quad n_{3} \neq n_{1} \quad n_{4}=6-n_{3} \quad n_{5}=n_{3} \quad n_{6}=n_{4} \\
& d=\frac{1}{2}\left(5-6 n_{1}+n_{1}^{2}\right) \quad e=5-d-6 n_{3}+n_{3}^{2} .
\end{aligned}
$$

There are four admissible choices of $n_{1}, n_{3}$. The cases $n_{1}=2, n_{3}=1, n_{1}=3, n_{3}=1$ and $n_{1}=3, n_{3}=2$ lead to inconsistent recursion relations for the coefficients of (2.1). In the case $n_{1}=1, n_{3}=2$, when $a=-6, b=d=0, c=e=-3$, we have to set $f=-2 s, g=0$ for the recursion relations to become consistent. Up to a scale transformation of variables, this is the case (1.8) of (1.6).

If $b=e$, we have

$$
(n+1) n^{3}(n-3)(n-4)\left(n^{2}-6 n+5-2 e\right)\left(n^{2}-6 n+5-d-e\right)^{2}=0
$$


i.e. six resonances lie in the positions $n=-1,0,0,0,3,4$. Denoting the positions of other six resonances as $n_{1}, n_{2}, \ldots, n_{6}$, we obtain

$$
\begin{aligned}
& n_{2}=6-n_{1} \quad n_{4}=6-n_{3} \quad n_{5}=n_{3} \quad n_{6}=n_{4} \\
& e=\frac{1}{2}\left(5-6 n_{1}+n_{1}^{2}\right) \quad d=5-e-6 n_{3}+n_{3}^{2} .
\end{aligned}
$$

There are six admissible choices of $n_{1}, n_{3}$. The cases $n_{1}=2, n_{3}=1, n_{1}=3, n_{3}=1$ and $n_{1}=3, n_{3}=2$ lead to inconsistent recursion relations for the coefficients of (2.1). In the case $n_{1}=1, n_{3}=1$, when $a=c=-6, b=d=e=0$, we have to set $f=g=-2 s$ to make the recursion relations consistent at the resonances, thus obtaining the case (1.9) of (1.6). The case $n_{1}=1, n_{3}=2$ with $a=-6, b=e=0, c=d=-3$, where we have to set $f=g=-2 s$, leads us to the case (1.10) of (1.6). The case $n_{1}=2, n_{3}=2$ with $a=-9 / 2, b=d=e=-3 / 2, c=-3$, where we have to set $f=g=-s$, leads us to the case $(1.11)$ of $(1.6)$.

2.2.2. Case $\alpha+\beta=-2, \gamma+\delta>-2$ We set $q_{0} \bar{q}_{0}=1$ without loss of generality. If $\alpha \neq \beta$ or $\gamma \neq \delta$, there are two or more resonances in negative positions. Therefore we set $\alpha=\beta=-1, \gamma=\delta>-1$ and obtain

$$
\begin{aligned}
& a=-6-b \quad d=-e+(2+c) \delta-3 \delta^{2}+\delta^{3} \\
& (n+1) n^{3}(n-3)(n-4)\left(n^{2}-6 n+5-2 b\right) \times \\
& \left(n^{2}-3(1-\delta) n+2+c-6 \delta+3 \delta^{2}\right)^{2}=0
\end{aligned}
$$

i.e. six resonances lie in the positions $n=-1,0,0,0,3,4$. Denoting the positions of other six resonances as $n_{1}, n_{2}, \ldots, n_{6}$, we find from (2.5) that $n_{2}=6-n_{1}, n_{1}=1,2,3$, $b=\frac{1}{2}\left(5-6 n_{1}+n_{1}^{2}\right), \delta=0, n_{3}=n_{5}=1, n_{4}=n_{6}=2, c=0$ and $d=-e$. Then we check the consistency of recursion relations at the resonances. In the case $n_{1}=1$, we have to set $e=g=0$ and $f=-2 s$ at $n=1$, and the system (1.6) becomes two independent Hirota equations [3]. In the case $n_{1}=2$, we have to set $e=g=0$ at $n=1$ and $f=-s$ at $n=3$, thus obtaining two independent Sasa-Satsuma equations [4, 5]. In the case $n_{1}=3$, the recursion relations are inconsistent at $n=3$.

\subsection{Singular generic branches: $h=0$}

When $h=0$, we set $s=1$ without loss of generality. The consideration of dominant terms of (1.6) leads us to the following two cases to be studied separately: $\alpha+\beta=$ $\gamma+\delta=-1$ and $\alpha+\beta=-1, \gamma+\delta>-1$ (for the reason of symmetry, we omit $\alpha+\beta>-1, \gamma+\delta=-1)$.

2.3.1. Case $\alpha+\beta=\gamma+\delta=-1$ In this case, we have two possibilities: either two or three of the resonances lie in the position $n=0$.

Two resonances at $\boldsymbol{n}=\mathbf{0}$. If both $q_{0} \bar{q}_{0}$ and $r_{0} \bar{r}_{0}$ are some fixed nonzero constants, then the recursion relations turn out to be inconsistent at the resonance $n=1$. 
Three resonances at $\boldsymbol{n}=\mathbf{0}$. We set $q_{0} \bar{q}_{0}=\mathrm{i}+\epsilon r_{0} \bar{r}_{0}$ w.l.g., and then find two possibilities: $\epsilon=-1$ and $\epsilon=1$.

When $\epsilon=-1$, we have

$$
\begin{aligned}
& a=\frac{-2-5 \alpha-5 \alpha^{2}}{1+2 \alpha} \quad b=e=\frac{-3 \alpha-3 \alpha^{2}}{1+2 \alpha} \quad d=a-c \quad \gamma=\alpha \\
& (n+1) n^{3}(n-2)(n-3)(n-1-k)(n-3+k)=0
\end{aligned}
$$

where $k=-c-2 \alpha$, and only $k=0,1,2$ are admissible. The case $k=2$ is related to $k=0$ through $q \leftrightarrow \bar{q}, r \leftrightarrow \bar{r}, x \rightarrow-x, t \rightarrow-t$. In the case $k=1$, the recursion relations are inconsistent at the resonances $n=2$. In the case $k=0$, we have to set $g=f$ at the resonance $n=1$, and then the recursion relations are consistent at $n=2,3$ if and only if $c=2, c=-2, c=0$ or $c=4$, which, after scale transformations of variables, give us the cases (1.12), (1.13), (1.14) and (1.15) of the system (1.6), respectively.

When $\epsilon=1$, we have

$$
\begin{aligned}
& a=\frac{-2-5 \alpha-5 \alpha^{2}}{1+2 \alpha} \quad b=-d=\frac{-3 \alpha-3 \alpha^{2}}{1+2 \alpha} \quad e=-a-c \quad \gamma=-1-\alpha \\
& (n+1) n^{3}(n-2)(n-3)(n-1-l)(n-3+l)=0
\end{aligned}
$$

where $l=c-2 \alpha$. In all the admissible cases $l=0,1,2$, however, the recursion relations are inconsistent at the resonance $n=2$.

2.3.2. Case $\alpha+\beta=-1, \gamma+\delta>-1 \quad$ We set $q_{0} \bar{q}_{0}=\mathrm{i}$ w.l.g., and then find the following two possibilities to have the resonances in admissible positions (see [24] for more details):

$$
\begin{aligned}
& a=\frac{-2-5 \alpha-5 \alpha^{2}}{1+2 \alpha} \quad b=\frac{-3 \alpha-3 \alpha^{2}}{1+2 \alpha} \\
& c=\frac{(1+2 \alpha) e+(1+\alpha)\left(\gamma-\gamma^{2}\right)-\alpha\left(\delta-\delta^{2}\right)}{(1+\alpha) \gamma+\alpha \delta} \quad d=\frac{(\alpha \gamma+(1+\alpha) \delta) e+\gamma \delta(2-\gamma-\delta)}{(1+\alpha) \gamma+\alpha \delta} \\
& (n+1) n^{3}(n-2)(n-3)\left(n+\gamma+\delta+\frac{(1+2 \alpha) e-(2 \alpha+\gamma) \delta}{(1+\alpha) \gamma+\alpha \delta}\right) \times \\
& \left(n+\gamma+\delta-2-\frac{(1+2 \alpha) e-(2 \alpha+\gamma) \delta}{(1+\alpha) \gamma+\alpha \delta}\right)=0 ; \\
& a=\frac{-2-5 \alpha-5 \alpha^{2}}{1+2 \alpha} \quad b=\frac{-3 \alpha-3 \alpha^{2}}{1+2 \alpha} \quad d=e=\gamma=\delta=0 \\
& (n+1) n^{3}(n-2)(n-3)(n-1+c)(n-1-c)=0 .
\end{aligned}
$$

In the case (2.6), taking into account that four of the resonances should lie in positive integer positions and that $\gamma, \delta$ should be integer, we have to set $e=$ $\delta-\delta^{2} /(1+2 \alpha)$ and $\gamma=-\delta$. Now the positions of resonances are $n=-1,0,0,0,1,1,2,3$. At $n=1$, we have to set $g=0, \delta=-1-2 \alpha, \alpha(\alpha+1)=0$, and then the recursion relations become consistent at $n=2,3$ as well. Both choices of $\alpha, \alpha=0$ and $\alpha=-1$, lead to the case $(1.16)$ of $(1.6)$.

In the case (2.7), we have to set $c=0$ to ensure admissible positions of resonances. Then we obtain $g=0$ at $n=1$, and the system (1.6) becomes a pair of uncoupled equations. 


\subsection{Other branches}

We have proven that the system (1.6) admits good singular generic branches in the cases (1.7)-(1.16) only. In each of those cases, however, the system (1.6) admits many other branches. They are Taylor expansions, which all are governed by the CauchyKovalevskaya theorem in the case of (1.6) and need no analysis, as well as singular nongeneric branches, which all have to be studied. All the singular non-generic branches of the cases (1.7)-(1.16) of (1.6) turn out to be good, in the sense that the exponents $\alpha, \beta, \gamma, \delta$ and the positions $n$ of resonances are integer and the recursion relations are consistent. Omitting here the lengthy consideration of all cases, we give as an illustration the following two singular non-generic branches of the case (1.7) (where $h=1, a=-3 / 2)$ :

(i) $\alpha=-1, \beta=-1, \gamma=-2, \delta=2, q_{0} \bar{q}_{0}=4$, positions of resonances are $n=-4,-1,0,0,0,1,1,3,4,4,5,5$;

(ii) $\alpha=-2, \beta=0, \gamma=-3, \delta=2, q_{0} \bar{q}_{0}=8$, positions of resonances are $n=-5,-2,-1,0,0,0,2,4,5,5,6,7$.

We can conclude now that the system (1.6) passes the Painlevé test for integrability only in the cases (1.7)-(1.16). Let us establish in the succeeding section that the system (1.6) in all the cases (1.7)-(1.16) possesses a Lax representation.

\section{Integrability}

\subsection{Known cases and new cases}

First of all, let us note that, by the transformation

$$
\begin{array}{lrl}
x \rightarrow x+\rho t & t \rightarrow t & \\
q \rightarrow q \exp (\mathrm{i} \omega) & \bar{q} \rightarrow \bar{q} \exp (-\mathrm{i} \omega) \\
r & \rightarrow r \exp (\mathrm{i} \omega) & \bar{r} \rightarrow \bar{r} \exp (-\mathrm{i} \omega) \\
\omega & =\sigma x+\tau t &
\end{array}
$$

with appropriately chosen real constants $\rho, \sigma, \tau$, we can make $s=f=g=0$ in the cases (1.7) $-(1.11)$ of $(1.6)$ and $f=g=0$ in the cases (1.12)-(1.16) of (1.6).

If we set $s=f=g=0$ in (1.8) and (1.10), these two cases of (1.6) turn out to be simply related by $q \rightarrow q, \bar{q} \rightarrow \bar{q}, r \rightarrow \bar{r}, \bar{r} \rightarrow r$. Since the case (1.10) is known to be integrable [25], the case (1.8) is integrable as well.

The integrability of the system (1.6) with (1.9), written in a form of coupled modified Korteweg-de Vries (mKdV) equations, was proven in [26] (see also [27]).

A Lax pair for the case (1.11) of (1.6) was given in [28].

The integrability of the system (1.6) with (1.13) was proven in [29].

The cases (1.12), (1.14) and (1.15) are integrable due to the work [30, 31].

The remaining two cases, (1.7) and (1.16), of the system (1.6) turn out to be new, and we prove their integrability in sections 3.2 and 3.3 , respectively. 


\subsection{Case (1.7)}

Without loss of generality, we set $h=-1, a=-6, s=0$ and study the case (1.7) of (1.6) in the form of four coupled $\mathrm{mKdV}$ equations

$$
\begin{aligned}
& q_{t}+q_{x x x}+6 q \bar{q} q_{x}+6(q r \bar{r})_{x}=0 \\
& \bar{q}_{t}+\bar{q}_{x x x}+6 q \bar{q} \bar{q}_{x}+6(\bar{q} r \bar{r})_{x}=0 \\
& r_{t}+r_{x x x}+6 r \bar{r} r_{x}+6(q \bar{q} r)_{x}=0 \\
& \bar{r}_{t}+\bar{r}_{x x x}+6 r \bar{r} \bar{r}_{x}+6(q \bar{q} \bar{r})_{x}=0 .
\end{aligned}
$$

The compatibility condition $U_{t}-V_{x}+U V-V U=0$ of the linear problem $\Psi_{x}=U \Psi, \Psi_{t}=$ $V \Psi$ becomes the system of two matrix mKdV equations 32,

$$
\begin{aligned}
& Q_{t}+Q_{x x x}-3 Q_{x} R Q-3 Q R Q_{x}=0 \\
& R_{t}+R_{x x x}-3 R_{x} Q R-3 R Q R_{x}=0
\end{aligned}
$$

if we take the matrices $U$ and $V$ in the following block form [26]:

$$
\begin{aligned}
& U= \mathrm{i} \zeta\left(\begin{array}{cc}
-I_{1} & 0 \\
0 & I_{2}
\end{array}\right)+\left(\begin{array}{cc}
0 & Q \\
R & 0
\end{array}\right) \\
& V=\mathrm{i} \zeta^{3}\left(\begin{array}{cc}
-4 I_{1} & 0 \\
0 & 4 I_{2}
\end{array}\right)+\zeta^{2}\left(\begin{array}{cc}
0 & 4 Q \\
4 R & 0
\end{array}\right)+\mathrm{i} \zeta\left(\begin{array}{cc}
-2 Q R & 2 Q_{x} \\
-2 R_{x} & 2 R Q
\end{array}\right)+ \\
& \\
&\left(\begin{array}{cc}
Q_{x} R-Q R_{x} & -Q_{x x}+2 Q R Q \\
-R_{x x}+2 R Q R & R_{x} Q-R Q_{x}
\end{array}\right)
\end{aligned}
$$

where $I_{1}$ and $I_{2}$ are unit matrices, $\zeta$ is a parameter. The choice

$$
Q=\left(\begin{array}{cc}
q & r \\
\bar{r} & \bar{q}
\end{array}\right) \quad R=-\left(\begin{array}{cc}
\bar{q} & r \\
\bar{r} & q
\end{array}\right)
$$

changes the system (3.2) into (3.1). This proves that the new case (1.7) of the coupled HONLS equations (1.6) possesses a parametric Lax pair. The existence of a Lax pair indicates that the system (3.1) is solvable under appropriate boundary conditions via the inverse scattering method. Indeed, we can solve the initial-value problem and obtain the $N$-soliton solution of (3.1) by imposing appropriate constraints on the matrix-valued scattering data [26].

Usually, it is straightforward to obtain a multi-field extension of integrable twocomponent systems based on the Lax formulation. In fact, we can obtain an $m$ component generalization of the two-component system (1.6) in the cases (1.8), (1.10) and (1.12)-(1.15) by replacing two-component vectors in the Lax pair with $m$-component vectors. For the case (1.11), we have only to replace four-component vectors in the Lax pair with $2 m$-component vectors (this is clear if we express the Sasa-Satsuma equation in the form of coupled $\mathrm{mKdV}$ equations [四]). However, a multi-field generalization of the case (1.7) as well as the case (1.9) is not straightforward. Thus we explain a multicomponent generalization of the system (3.1), which is analogous to that of the case 
(11.9) (cf [26]). For this purpose, we choose the matrices $Q$ and $R$ as

$$
\begin{aligned}
& Q=\left(\begin{array}{cc}
u_{0} I \otimes I+\sum_{k=1}^{2 m-1} u_{k} e_{k} \otimes I & v_{0} I \otimes I+\sum_{k=1}^{2 m-1} v_{k} I \otimes e_{k} \\
v_{0} I \otimes I-\sum_{k=1}^{2 m-1} v_{k} I \otimes e_{k} & u_{0} I \otimes I-\sum_{k=1}^{2 m-1} u_{k} e_{k} \otimes I
\end{array}\right) \\
& R=-\left(\begin{array}{cc}
u_{0} I \otimes I-\sum_{k=1}^{2 m-1} u_{k} e_{k} \otimes I & v_{0} I \otimes I+\sum_{k=1}^{2 m-1} v_{k} I \otimes e_{k} \\
v_{0} I \otimes I-\sum_{k=1}^{2 m-1} v_{k} I \otimes e_{k} & u_{0} I \otimes I+\sum_{k=1}^{2 m-1} u_{k} e_{k} \otimes I
\end{array}\right)
\end{aligned}
$$

where $I$ is the $2^{m-1} \times 2^{m-1}$ unit matrix, and $\left\{e_{1}, \ldots, e_{2 m-1}\right\}$ are $2^{m-1} \times 2^{m-1}$ anticommutative and anti-Hermitian matrices:

$$
\left\{e_{i}, e_{j}\right\}_{+}=-2 \delta_{i j} I \quad e_{k}^{\dagger}=-e_{k} .
$$

Then the matrix mKdV equations (3.2) become the system

$$
\begin{aligned}
& u_{j, t}+u_{j, x x x}+6 \sum_{k=0}^{2 m-1} u_{k}^{2} u_{j, x}+6\left(\sum_{k=0}^{2 m-1} v_{k}^{2} u_{j}\right)_{x}=0 \\
& v_{j, t}+v_{j, x x x}+6 \sum_{k=0}^{2 m-1} v_{k}^{2} v_{j, x}+6\left(\sum_{k=0}^{2 m-1} u_{k}^{2} v_{j}\right)_{x}=0
\end{aligned}
$$

Assuming that $u_{k}$ and $v_{k}$ are real and setting

$$
\begin{aligned}
& u_{2 j-2}+\mathrm{i} u_{2 j-1}=q_{j} \\
& v_{2 j-2}+\mathrm{i} v_{2 j-1}=r_{j}
\end{aligned} \quad j=1,2, \ldots, m
$$

we obtain a multi-component generalization of (3.1):

$$
\begin{aligned}
& q_{j, t}+q_{j, x x x}+6 \sum_{k=1}^{m}\left|q_{k}\right|^{2} q_{j, x}+6\left(\sum_{k=1}^{m}\left|r_{k}\right|^{2} q_{j}\right)_{x}=0 \\
& r_{j, t}+r_{j, x x x}+6 \sum_{k=1}^{m}\left|r_{k}\right|^{2} r_{j, x}+6\left(\sum_{k=1}^{m}\left|q_{k}\right|^{2} r_{j}\right)_{x}=0
\end{aligned} \quad j=1,2, \ldots, m .
$$

\subsection{Case (1.10)}

Without loss of generality, we set $a=2, s=1, f=0$ and study the case (1.16) of (1.6) in the form

$$
\begin{aligned}
& q_{t}=\mathrm{i} q_{x x}+2\left(q \bar{q} q_{x}+r \bar{r} q_{x}+q r \bar{r}_{x}\right) \\
& r_{t}=\mathrm{i} r_{x x}+2\left(r \bar{r} r_{x}+q \bar{q} r_{x}+r q \bar{q}_{x}\right) .
\end{aligned}
$$

Using the Mathematica package condens.m [33], we can check that the system (3.3) has two conservation laws for each rank from 1 to (at least) 4 . The fact, that the conservation laws appear by pairs, is highly suggestive that equations (3.3) can be uncoupled by some transformation. Using the first conservation laws of (3.3),

$$
\begin{aligned}
& (q \bar{q})_{t}=\left\{\mathrm{i}\left(q_{x} \bar{q}-q \bar{q}_{x}\right)+(q \bar{q})^{2}+2 q \bar{q} r \bar{r}\right\}_{x} \\
& (r \bar{r})_{t}=\left\{\mathrm{i}\left(r_{x} \bar{r}-r \bar{r}_{x}\right)+(r \bar{r})^{2}+2 r \bar{r} q \bar{q}\right\}_{x}
\end{aligned}
$$

and introducing the new dependent variables by

$$
q=u \exp \left(\mathrm{i} \int_{x_{0}}^{x} v \bar{v} \mathrm{~d} x^{\prime}\right) \quad r=v \exp \left(\mathrm{i} \int_{x_{0}}^{x} u \bar{u} \mathrm{~d} x^{\prime}\right)
$$


we find that the system (3.3) is equivalent to two independent Chen-Lee-Liu equations [6]:

$$
u_{t}=\mathrm{i} u_{x x}+2 u \bar{u} u_{x} \quad v_{t}=\mathrm{i} v_{x x}+2 v \bar{v} v_{x}
$$

(here we have assumed that the dependent variables approach zero as $x \rightarrow x_{0}$ ). Since a Lax pair for the Chen-Lee-Liu equation is well-known, we can straightforwardly obtain a Lax pair with two spectral parameters for the system (3.3) by using a gauge transformation. This proves the integrability of the new case (1.16) of (1.6). It is remarkable that, due to (3.4), the relations $|q|=|u|,|r|=|v|$ are satisfied. These relations show that solitons observed in channel $q$ and solitons observed in channel $r$ interact only in their phase parts.

\section{Concluding remarks}

In this paper, we have carried out the singularity analysis of a system of two symmetrically coupled higher-order nonlinear Schrödinger (HONLS) equations with sufficient arbitrariness of the coefficients. Apparently, the form of coupled HONLS equations (1.6) is more general than those which have been studied by means of the Painlevé analysis 17, 18]. Assuming the general form of coupled HONLS equations, we have exhaustively selected as many as ten cases in which the system passes the Painlevé test for integrability well. The integrability of eight cases in the Lax sense has been already known, while the remaining two cases turned out to be new. We have verified the integrability of the two cases by considering a reduction of integrable matrix equations and a nonlinear transformation into uncoupled integrable equations, respectively. The work developed in this paper corresponds to the spirit of the work [19] by Sofia Kovalevskaya, who originated the singularity analysis of nonlinear physical systems.

We should comment on the result obtained in this paper. First, though the form of coupled HONLS equations (1.6) is general enough within limitations of computer's performance, we may obtain new integrable systems of coupled HONLS equations by assuming a more general form. Secondly, comparing the integrable cases (1.2)(1.5) of the single-component system with the integrable cases (1.7)-(1.16) of the twocomponent system, we notice that integrable one-component equations often have plural multi-component generalizations. We cannot identify each of (1.7)-(1.16) with a multicomponent generalization of only one of (1.2)-(1.5). For instance, the case (1.7) admits the reduction $r \rightarrow 0$ to the Hirota case (1.2) as well as the reduction $r \rightarrow q$ to the Sasa-Satsuma case (1.3). Thirdly, study of exact solutions, which contain sufficiently many parameters, for integrable coupled HONLS equations has not been well-developed so far and deserve further investigation. 


\section{Acknowledgments}

S. Yu. S. is grateful for support to the National Fund for Fundamental Research of Belarus. T. T. appreciates a Research Fellowship of the Japan Society for the Promotion of Science for Young Scientists.

\section{References}

[1] Kodama Y 1985 J. Stat. Phys. 39597

[2] Kodama Y and Hasegawa A 1987 IEEE J. Quantum Electron. QE-23 510

[3] Hirota R 1973 J. Math. Phys. 14805

[4] Yajima N and Oikawa M 1975 Prog. Theor. Phys. 541576

[5] Sasa N and Satsuma J 1991 J. Phys. Soc. Japan 60409

[6] Chen H H, Lee Y C and Liu C S 1979 Phys. Scr. 20490

[7] Kaup D J and Newell A C 1978 J. Math. Phys. 19798

[8] Clarkson P A and Cosgrove C M 1987 J. Phys. A: Math. Gen. 202003

[9] Porsezian K and Nakkeeran K 1996 Phys. Rev. Lett. 763955

[10] Mihalache D, Truta N and Crasovan L-C 1997 Phys. Rev. E 561064

[11] Gedalin M, Scott T C and Band Y B 1997 Phys. Rev. Lett. 78448

[12] Sakovich S Yu 1997 J. Phys. Soc. Japan 662527

[13] Nijhof J H B and Roelofs G H M 1992 J. Phys. A: Math. Gen. 252403

[14] Agrawal G P 1995 Nonlinear Fiber Optics 2nd edn (San Diego: Academic Press)

[15] Sahadevan R, Tamizhmani K M and Lakshmanan M 1986 J. Phys. A: Math. Gen. 191783

[16] Park Q-H and Shin H J 1999 Phys. Rev. E 592373

[17] Porsezian K, Shanmugha Sundaram P and Mahalingam A 1994 Phys. Rev. E 501543

[18] Radhakrishnan R, Lakshmanan M and Daniel M 1995 J. Phys. A: Math. Gen. 287299

[19] Kovalevskaya S 1889 Acta Mathematica 12177

[20] Weiss J, Tabor M and Carnevale G 1983 J. Math. Phys. 24522

[21] Jimbo M, Kruskal M D and Miwa T 1982 Phys. Lett. A 9259

[22] Wolfram S 1999 The Mathematica Book 4th edn (Cambridge: Cambridge University Press)

[23] Sakovich S Yu and Tsuchida T 2000 Preprint arXiv nlin.SI/0002023

[24] Sakovich S Yu and Tsuchida T 2000 Preprint arXiv nlin.SI/0004025

[25] Tasgal R S and Potasek M J 1992 J. Math. Phys. 331208

[26] Tsuchida T and Wadati M 1998 J. Phys. Soc. Japan 671175

[27] Svinolupov S I and Sokolov V V 1994 Theor. Math. Phys. 100959

[28] Nakkeeran K, Porsezian K, Shanmugha Sundaram P and Mahalingam A 1998 Phys. Rev. Lett. 80 1425

[29] Morris H C and Dodd R K 1979 Phys. Scr. 20505

[30] Tsuchida T and Wadati M 1999 Phys. Lett. A 25753

[31] Tsuchida T and Wadati M 1999 Inverse Problems 151363

[32] Athorne C and Fordy A 1987 J. Phys. A: Math. Gen. 201377

[33] Göktaş Ü and Hereman W 1997 J. Symbolic Comput. 24591 